\title{
Activity of hippocampal adult-born neurons regulates alcohol withdrawal seizures
}

\author{
Daehoon Lee, ${ }^{1}$ Balu Krishnan, ${ }^{2}$ Hai Zhang, ${ }^{1}$ Hee Ra Park, ${ }^{1}$ Eun Jeoung Ro, ${ }^{1}$ Yu-Na Jung, ${ }^{3}$ \\ and Hoonkyo Suh ${ }^{1}$ \\ 'Department of Neurosciences and ${ }^{2}$ Epilepsy Center, Neurological Institute, Cleveland Clinic, Cleveland, Ohio, USA. \\ ${ }^{3}$ Department of Biochemistry, Case Western Reserve University, Cleveland, Ohio, USA
}

\begin{abstract}
Alcohol withdrawal (AW) after chronic alcohol exposure produces a series of symptoms, with AW-associated seizures being among the most serious and dangerous. However, the mechanism underlying AW seizures has yet to be established. In our mouse model, a sudden AW produced 2 waves of seizures: the first wave includes a surge of multiple seizures that occurs within hours to days of AW, and the second wave consists of sustained expression of epileptiform spikes and wave discharges (SWDs) during a protracted period of abstinence. We revealed that the structural and functional adaptations in newborn dentate granule cells (DCCs) in the hippocampus underlie the second wave of seizures but not the first wave. While the general morphology of newborn DCCs remained unchanged, AW increased the dendritic spine density of newborn DCCs, suggesting that AW induced synaptic connectivity of newborn DCCs with excitatory afferent neurons and enhanced excitability of newborn DGCs. Indeed, specific activation and suppression of newborn DCCs by the chemogenetic DREADD method increased and decreased the expression of epileptiform SWDs, respectively, during abstinence. Thus, our study unveiled that the pathological plasticity of hippocampal newborn DGCs underlies AW seizures during a protracted period of abstinence, providing critical insight into hippocampal neural circuits as a foundation to understand and treat AW seizures.
\end{abstract}

Conflict of interest: The authors have declared that no conflict of interest exists.

Copyright: (c) 2019, American Society for Clinical Investigation.

Submitted: March 13, 2019

Accepted: August 31, 2019

Published: October 3, 2019.

Reference information: /CI Insight. 2019;4(19):e128770.

https://doi.org/10.1172/jci.

insight.128770.

\section{Introduction}

Chronic alcohol exposure disrupts brain homeostasis and progressively transforms the brain into a state of alcohol dependence $(1,2)$. Abrupt alcohol withdrawal (AW) in alcohol-dependent individuals results in the emergence of a series of physiological and psychological symptoms that are collectively referred to as alcohol withdrawal syndrome (AWS) (3-7). Clinical features of AWS include alcoholic hallucinosis, cognitive and emotional impairments, delirium tremens, and brain hyperexcitability (8-12). More than 2 million Americans are estimated to develop AWS each year $(13,14)$. Despite such negative effects of alcohol on physical and mental function, its positive-reinforcing effects such as euphoria and pain reduction enhances susceptibility to relapse during a withdrawal period (abstinence), and the repeated cycles of alcohol consumption and withdrawal are ultimately thought to lead to alcohol addiction (15-18). To prevent enhanced vulnerability to relapse and perpetual alcohol use, it is imperative to understand the mechanisms underlying AWS.

Generalized tonic-clonic seizures are the most dangerous and serious component of AWS, and they contribute to significant mortality of alcoholics (19-24). Alcohol is a well-known CNS depressant that facilitates and reduces inhibitory and excitatory signals, respectively, and chronic alcohol exposure induces prolonged and enhanced sedative effects (25). The brain attempts to counterbalance the depressant effect of alcohol by enhancing excitatory signals and decreasing inhibitory signals. However, continuous exposure to alcohol progressively dysregulates the neurophysiological system beyond normal homeostatic limits, and neuroadaptation that is masked by chronic exposure to alcohol is unveiled upon a sudden AW. This neuroadaptation, or brain allostatic state induced by chronic alcohol exposure, has been thought to underlie the emergence and expression of hyperexcitability of the brain and AW seizures when alcohol consumption ceases $(6,16,17)$; however, the precise neural substrates that undergo neuroadaptation during alcohol exposure and withdrawal are not yet understood. 
Interestingly, chronic alcohol exposure produced 2 waves of seizures upon AW in our mouse model. The first wave consisted of a surge of multiple seizures and epileptic spikes that occurred within hours of AW and lasted only for a few days during abstinence. In the second wave, epileptiform spikes and wave discharges (SWDs) were expressed for a protracted period of abstinence. In this study, we revealed that AW induces structural and functional changes in hippocampal newborn dentate granule cells (DGCs) and that neuroadaptation in newborn DGCs directly underlies AW seizures during the second wave of seizures. Consistent with the occurrence of epileptiform SWDs, AW increased dendric spine density and enhanced connectivity of newborn DGCs with excitatory afferent neurons. Furthermore, Designer Receptors Exclusively Activated By Designer Drugs-mediated (DREADD-mediated) chemogenetic analysis showed that specific activation and inhibition of hippocampal newborn neurons increased and decreased the expression of epileptiform SWD, respectively, during a protracted abstinence. Thus, our study reveals that chronic alcohol exposure induces aberrant neural circuits that facilitate hyperexcitability of the brain, and it reveals that the activity of hippocampal newborn DGCs directly regulates the expression of epileptiform SWDs during a protracted period of abstinence.

\section{Results}

$A W$ produces seizures and epileptic spikes. Mice had voluntary access to a Lieber-DeCarli liquid diet in which the same portion of carbohydrates was supplemented with either ethanol (alcohol-fed group) or dextrose (control pair-fed group) (26). As a result, both control and alcohol-fed mice consumed the same amount of calories during the experimental period (ref. 27 and Supplemental Figure 1A; supplemental material available online with this article; https://doi.org/10.1172/jci.insight.128770DS1). During the 4-week alcohol feeding period, mice consumed alcohol daily $(20.2 \pm 1.53 \mathrm{~g} / \mathrm{kg} /$ day $)$ and accumulated a significant amount of alcohol (545.0 $\pm 1.53 \mathrm{~g} / \mathrm{kg}$ ) (Supplemental Figure 1B). Alcohol-fed mice showed significantly higher peak blood alcohol concentrations (BACs) compared with control mice $(P<0.0001)$ when BAC was measured at the end of the third week of alcohol treatment (Supplemental Figure 1C). This average value $(0.395 \%)$ corresponds to 5 times higher than the legal alcohol limit for driving $(0.08 \%)$. Importantly, both alcohol-fed and control mice gained comparable weight during the alcohol treatment period ( $P=0.8731$ ) (Supplemental Figure 1D), indicating that both groups consumed similar amounts of calories during chronic alcohol exposure.

To investigate whether AW induces seizures, electroencephalograms (EEGs) were monitored in the cortex and hippocampus immediately after 4 weeks of alcohol exposure (Supplemental Figure 2 and Figure 1A). Upon AW, only alcohol-fed mice showed seizure activities and associated epileptic spikes, while both were completely absent in control mice (Figure 1B). Multiple seizure activities were observed within 24 hours of abstinence in most of the alcohol-fed mice (Figure 1, C and D). These seizure activities lasted longer than 30 seconds and always synchronized with the expression of seizure behavior as monitored by continuous video surveillance (Figure 1E). Characteristic epileptic spikes were observed only in alcohol-fed mice - not in control mice (Figure $1 \mathrm{~F}$ ). The frequencies of seizures and epileptic spikes did not correlate with the amount of alcohol consumption (Figure 1, G and $\mathrm{H}$ ).

$A W$ seizures are sustained during a protracted period of abstinence. After the disappearance of the first wave of epileptic seizures and spikes, our longitudinal EEG monitoring method identified unexpected sustained seizure activities for a prolonged period of abstinence, and this activity was completely absent from control mice (main effect: alcohol, $P<0.0001$ ) (Figure 2, A-D). Because only a few tonic-clonic seizures were detected during a protracted period of abstinence, we referred to spikes observed during the second wave of seizures as epileptiform SWDs. Interestingly, epileptiform SWDs changed in a time-dependent manner during a protracted abstinence (main effect: time, $P<0.0001$ ) (Figure 2D); alcohol-fed mice displayed epileptiform SWDs for up to 6 weeks of abstinence, while control mice did not show any signs of these spikes. Starting from 1 week of abstinence, the frequency of spikes gradually increased, peaked at 4 weeks of abstinence, and was abolished by 8 weeks of abstinence in alcohol-fed mice. The occurrence of epileptiform SWDs almost always synchronized between the hippocampus and cortex, suggesting generalized expression of epileptiform SWDs in the brain (Figure 2, B and C).

$A W$ increases the spine density of newborn DGCS. Our previous study showed that chronic alcohol exposure disrupted the synaptic connectivity of hippocampal newborn DGCs, dramatically reducing the density of dendritic spines of newborn DGCs (27). Here, we examined whether AW induces structural plasticity and impacts the function of newborn DGCs during a protracted abstinence. First, CAG-GFP retrovirus 


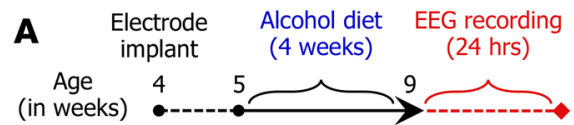

B

Control
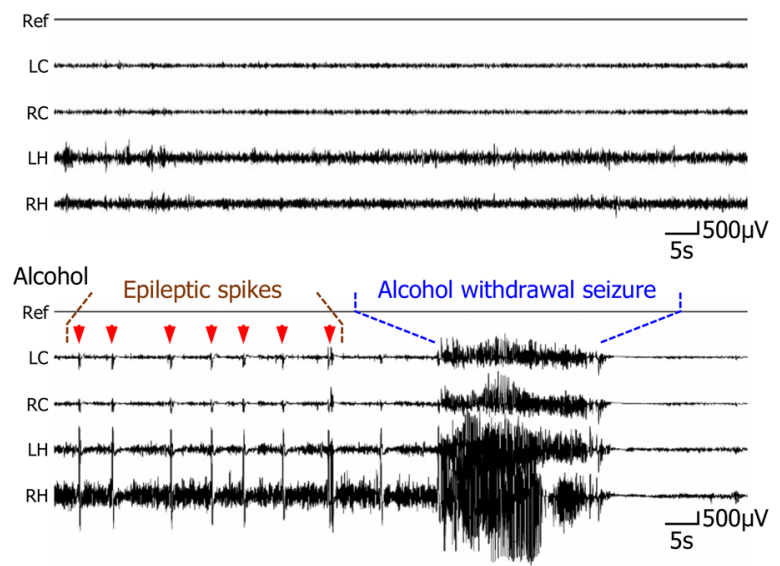

Figure 1. Alcohol withdrawal produces epileptic seizures. (A) Experimental timeline describing the alcohol feeding and electroencephalogram (EEG) recording schedule. (B) Representative EEG traces of control diet-fed (upper) and alcohol-fed (lower) mice upon alcohol withdrawal (AW). (C) Quantification of seizure frequency induced by AW $(2.33 \pm 1.86, n=6)$. (D) Distribution of seizure events within 24 hours of AW. (E) Average AW seizure duration (31.79 \pm 5.45 seconds, $n=14$ ). Seizure activities were absent in control mice. (F) Quantification of epileptic spikes within 24 hours of AW $(P<0.01$, alcohol-fed mice, $1442 \pm 687, n=6$; pair-fed mice, $25.0 \pm 10.17, n=4$ ). (G and $\mathbf{H})$ No correlation between alcohol consumption and frequencies of AW seizures and spikes was observed. ${ }^{* *} P$ $<0.01$ as determined by 2 -tailed unpaired $t$ tests. Data are represented as mean $\pm \mathrm{SD}$. Ref, reference; LC, left cortex; RC, right cortex; LH, left hippocampus; $\mathrm{RH}$, right hippocampus.
C
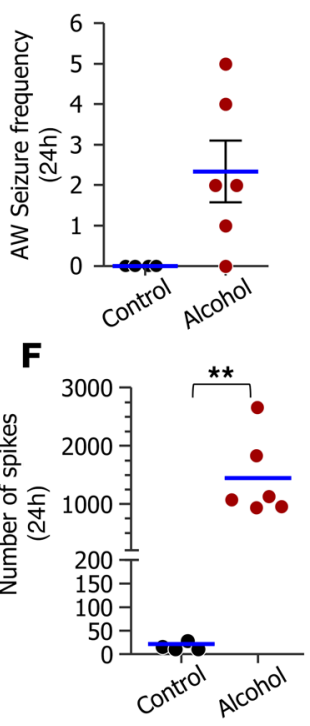

D

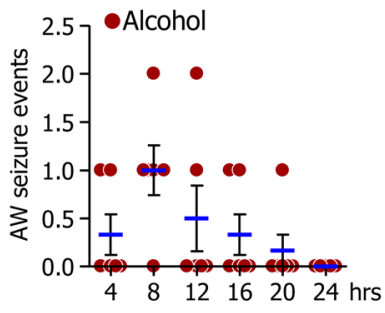

G $\quad R^{2}=0.04229$

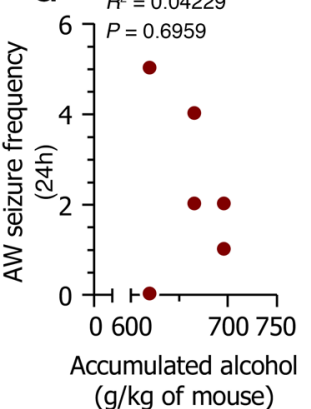

E

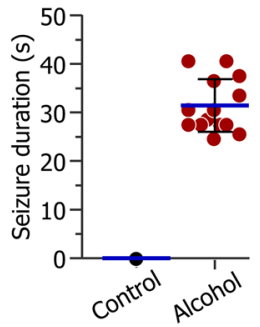

H

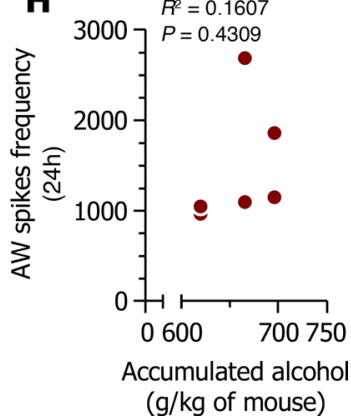

(RV-GFP) was injected into the dentate gyrus of the hippocampus to label newborn DGCs (28), and mice were exposed to alcohol for 4 weeks. The structural development of GFP-labeled newborn DGCs was examined at 2, 4, and 8 weeks of abstinence (Figure 3A). During abstinence, newborn DGCs did not show gross morphological changes; both control and alcohol-exposed mice showed comparable total dendritic lengths and numbers of dendritic branches in newborn DGCs during abstinence (Figure 3B). However, spine density was significantly increased in newborn DGCs of alcohol-fed mice compared with control mice at 2 and 4 weeks of abstinence, but not at 8 weeks of abstinence (Figure 3, C and D). Because excitatory neurons from the lateral entorhinal cortex (EC), medial EC, and hilus project to the distal, medial, and proximal dendrites of newborn DGCs in a topological manner (29), we examined the spine density of newborn DGCs in the outer, middle, and inner segments of dendrites. AW increased the spine density of newborn DGCs in all 3 segments of dendrites, indicating that AW globally induced synaptic connectivity with excitatory neurons at 2 and 4 weeks of abstinence, which are time points when alcohol-fed mice showed increased expression of SWDs (Figure 3, C and D).

Next, to determine whether AW induced the formation of different types of spines, we examined the densities of mushroom, stubby, and thin spines in the distal, medial, and proximal dendritic segments of 
A Electrode Alcohol diet implant (4 weeks) EEG recording

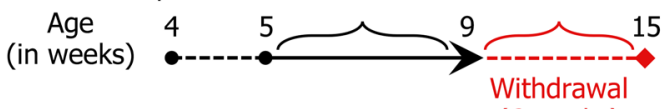

(6 weeks)

\section{B}

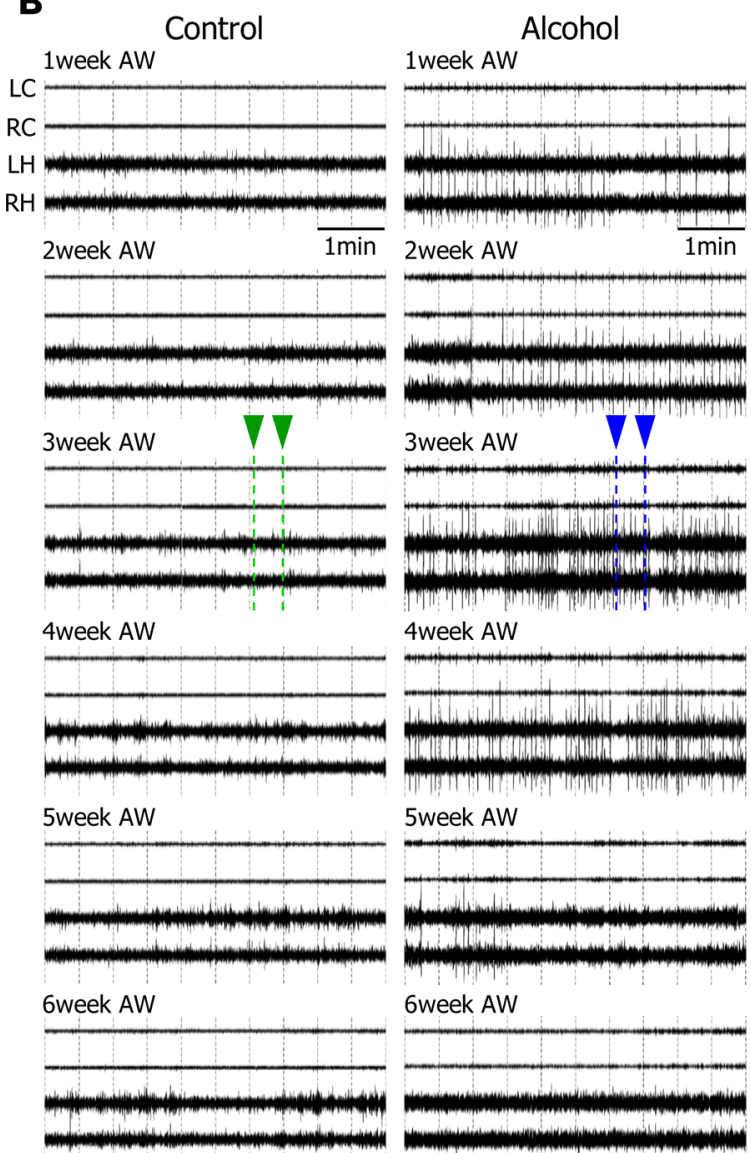

C
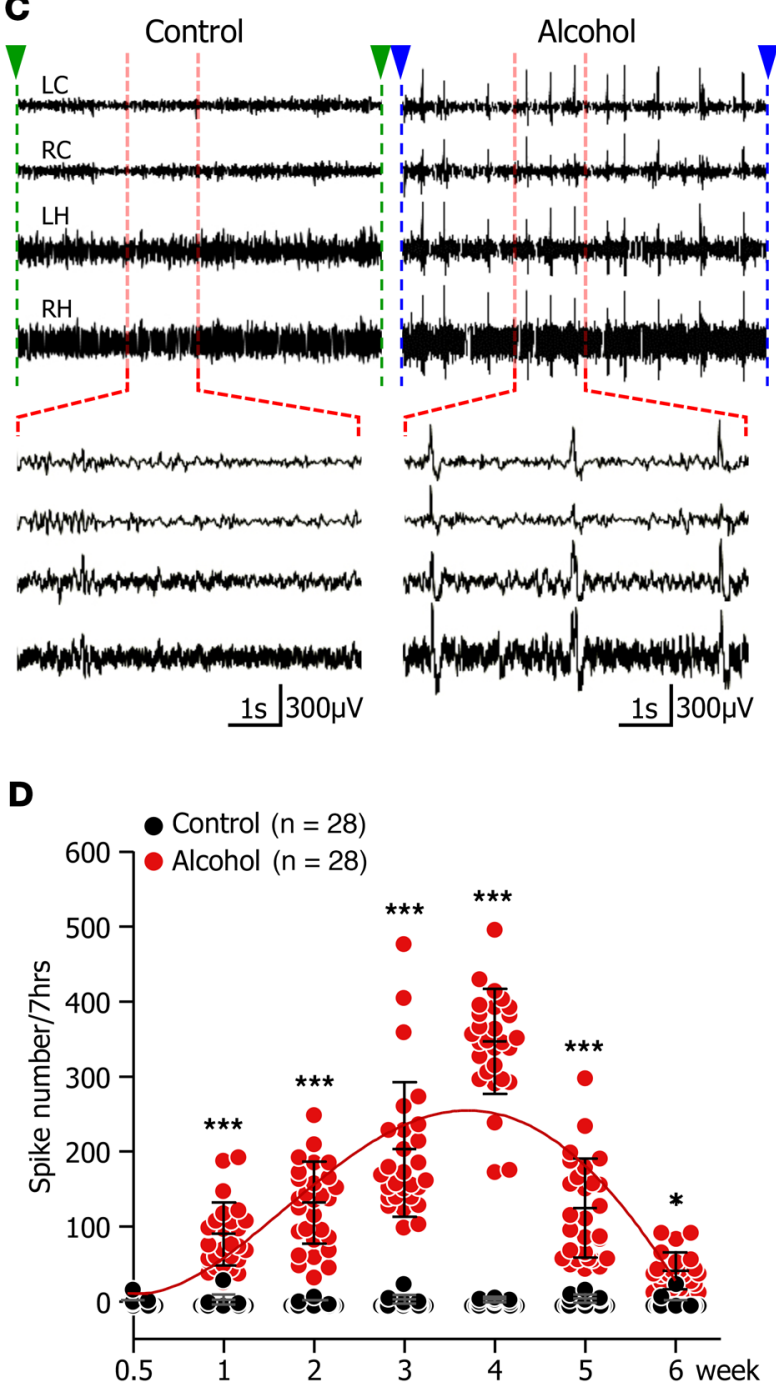

Figure 2. Sustained expression of spikes and wave discharges (SWDs) during a protracted period of abstinence. (A) Experimental timeline describing alcohol feeding and EEG recording schedule. (B) Representative images show the development of epileptiform SWDs during 6 weeks of abstinence. (C) Magnified images present the detailed shape and pattern of SWDs of pair-fed (green arrowheads) and alcohol-fed (blue arrowheads) mice. (D) Quantitative results show the development and progression of epileptiform SWDs during abstinence. Only alcohol-fed mice developed epileptiform SWDs during a 6-week abstinence (2-way repeated measures ANOVA: alcohol affect, $F_{1.324}=920.11, P<0.0001$ ), and the frequency of epileptiform SWDs changed over time (2-way repeated measures ANOVA: time effect, $F_{6,324}=117.2, P<0.0001$ ). ${ }^{*} P<0.05$; ${ }^{* * *} P<0.001$ as determined by Bonferroni post hoc tests showing significant differences in epileptiform SWDs between alcohol- and pair-fed control mice at a given time during abstinence. Data are represented as mean \pm SD. LC, left cortex; RC, right cortex; LH, left hippocampus; RH, right hippocampus.

newborn DGCs (Figure 3E). At 2 and 4 weeks of abstinence, AW significantly increased the densities of mushroom spines throughout dendritic segments of newborn DGCs, while the densities of thin spines remained unchanged (Figure $3 \mathrm{E}$ ). The densities of stubby spines were reduced in the medial and proximal segments, as well as in the medial segment of dendrites at 2 and 4 weeks of abstinence, respectively.

Specific inhibition of newborn DGCS suppresses the expression of SWDs during abstinence. To test the requirement of newborn DGCs for the expression of AW seizures, we specifically inhibited neuronal activity of newborn DGCs by using a DREADD method (30-32). First, we tested whether the induction of the first wave of seizures was dependent upon newborn DGCs. Tamoxifen was injected into NCE; hM4Difl/fl mice (NCE-M4), and then mice were exposed to alcohol for 4 weeks. During the first 24 hours of AW, clozapine N-oxide (CNO, $10 \mathrm{mg} / \mathrm{kg}$ ) was administered to both NCE-M4 and control mice a total of 6 times at 4-hour intervals. Tamoxifen injections successfully induced the expression of inhibitory DREADD receptors in newborn NCE-M4 mice, but not in control mice (M4), as determined by immunostaining against 
an HA tag that was conjugated to hM4Di receptors (Figure 4, A and B). However, CNO administration did not change the number of spikes $(P=0.9225)$, the seizure frequency $(\mathrm{P}>0.9999)$, or seizure duration $(P=0.9707)$ in either NCE-M4 or control mice (Figure 4, C-E), indicating that the specific inhibition of newborn DGCs did not impact the induction of the first wave of AW seizures.

Second, in order to investigate the role of hippocampal newborn DGC activity in the expression of the second wave of seizures, tamoxifen was injected into both NCE-M4 and control mice, and mice were exposed to alcohol for 4 weeks. Then, the effects of CNO on the expression of epileptiform SWDs was examined weekly using EEG monitoring (Figure 4F). AW after chronic alcohol exposure produced epileptiform SWDs in both control and NCE-M4 (Figure 4F). However, CNO injection significantly reduced the frequency of epileptiform SWDs only in NCE-M4 DREADD mice and not in control mice, indicating that specific inhibition of newborn DGCs reduced the expression of epileptiform SWDs at $2(P<0.001)$, $3(P<0.001)$, and $4(P<0.001)$ weeks of abstinence (Figure $4 \mathrm{~F})$. In pair-fed control groups, both control (non-DREADD) and NCE-M4 DREADD mice showed neither epileptiform SWDs nor any response to CNO administration. Importantly, the DREADD-mediated decrease in epileptiform SWDs is inducible and reversible, as epileptiform SWDs returned to basal levels when CNO was no longer available in the system (Supplemental Figure 3 and ref. 32).

Specific activation of newborn DGCs increases the expression of epileptiform SWDs during abstinence. To determine whether increased neuronal activity of hippocampal DGCs is sufficient to induce the expression of epileptiform SWDs during abstinence, we used NCE;hM3Dq ${ }^{\mathrm{f} / \mathrm{fl}}$ (NCE-M3) mice to express an excitatory DREADD receptor, hM3Dq, in newborn DGCs (33). Tamoxifen was injected into 4-week-old mice, mice were exposed to alcohol for 4 weeks, and electrodes were implanted to monitor the development of epileptiform SWDs during abstinence (Figure 5A). Equivalent numbers of hM3Dq receptors were expressed in newborn DGCs of NCE-M3 mice in both pair- and alcohol-fed groups as determined by a mCitrine reporter expression coexpressed with $\mathrm{hM} 3 \mathrm{Dq}$ (Figure 5A). Importantly, a CNO dose titration experiment showed that $0.8 \mathrm{mg} / \mathrm{kg}$ of CNO was sufficient to induce epileptiform SWDs in NCE-M3 mice only in the alcohol group and not in the nonalcohol group (Figure 5B), and this dose of CNO was subsequently used throughout the experiments. When AW produced epileptiform SWDs in alcohol-fed mice, CNO administration significantly increased the expression of epileptiform SWDs only in NCE-M3 mice and not in control mice, and these CNO-mediated increased epileptiform SWDs were observed at $2(P<0.001), 3(P<0.001)$, $4(P<0.01)$, and $5(P<0.01)$ weeks of abstinence (Figure $5 C)$. In control mice that were not exposed to alcohol, neither naive nor NCE-M3 mice displayed epileptiform SWDs in response to CNO (Figure 5C). The expression of epileptiform SWDs was tightly regulated by the action of CNO and DREADD receptors, and the frequency of epileptiform SWDs returned to basal levels when CNO was no longer available in NCE-M3 mice (Supplemental Figure 4).

\section{Discussion}

In response to continued excessive alcohol exposure, the brain institutes neuroadaptation in order to counterbalance the effects of the alcohol; however, the constant challenges presented by chronic alcohol consumption surpass the brain's ability to restore equilibrium, resulting in the development of an allostatic state, namely alcohol tolerance and dependence. Importantly, this allostatic state is manifested by the emergence and expression of deficits in cognition and emotion, as well as physiological impairments such as seizures, upon AW $(16,17)$. Despite alcohol's aversive effects on mental and physical health $(34,35)$, its positive-reinforcing effects of euphoria, anxiolysis, and pain reduction dramatically increase the vulnerability to relapse and alcohol abuse (36). The severity and susceptibility to relapse and perpetuation of alcohol abuse underscores the urgent need to understand mechanisms underlying alcohol dependence and withdrawal in order to develop new therapeutic strategies to intervene and treat AW-associated syndromes.

Our study identified newborn DGCs as neural substrates that undergo neuroadaptation and contribute to the development of the allostatic state of the brain in response to alcohol exposure and withdrawal. Chronic alcohol exposure altered the structure and function of hippocampal newborn DGCs, and these changes underlie the emergence and expression of seizures and epileptic spikes/epileptiform SWDs during a protracted period of abstinence. Hippocampal DGCs are principal excitatory neurons that are continuously generated and integrated into neural circuits, and this process of neurogenesis provides plasticity to brain function and dysfunction (37-39). Disrupted neurogenesis has been implicated in addiction, deficits in cognition, and 

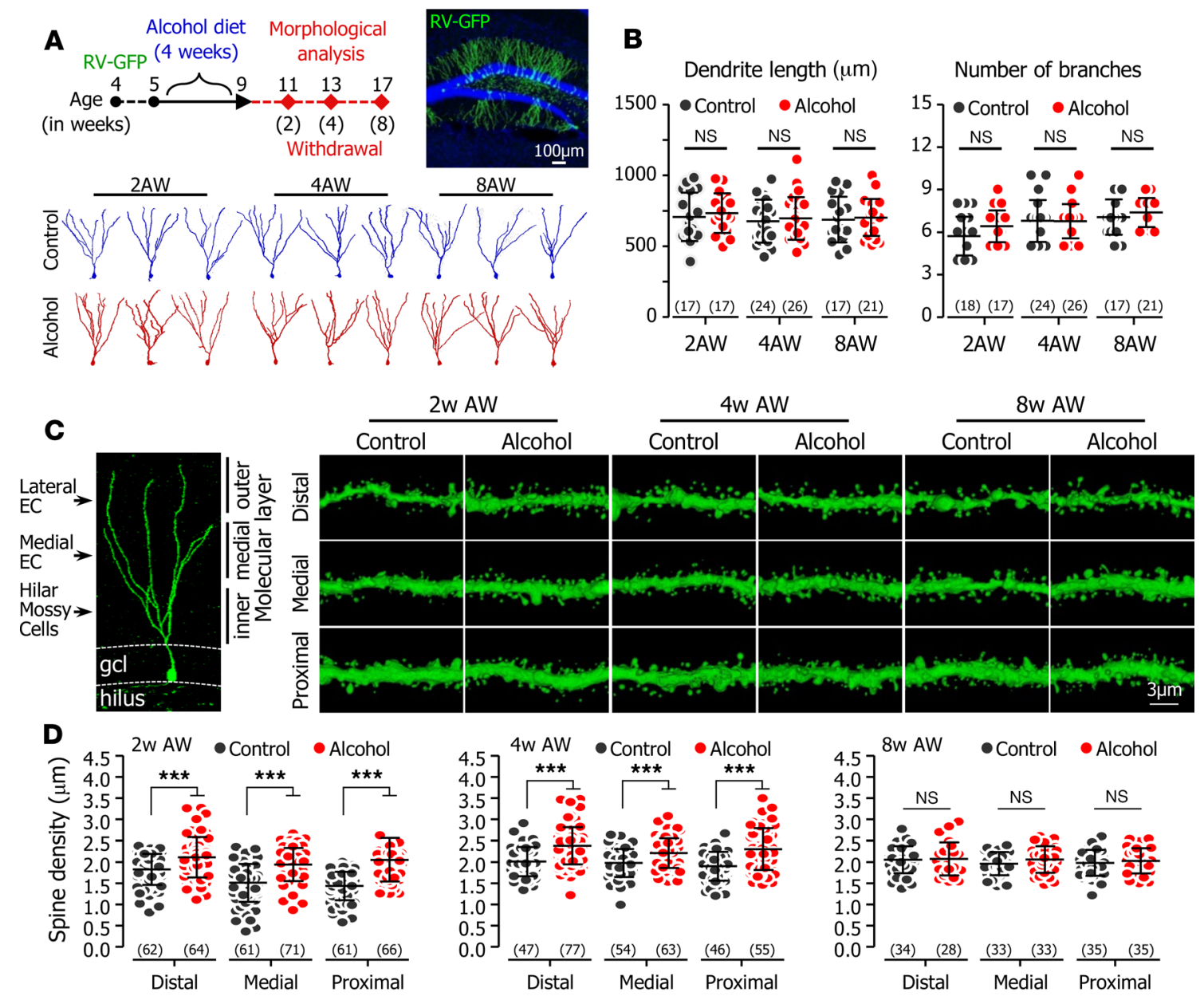

$\mathbf{E}$
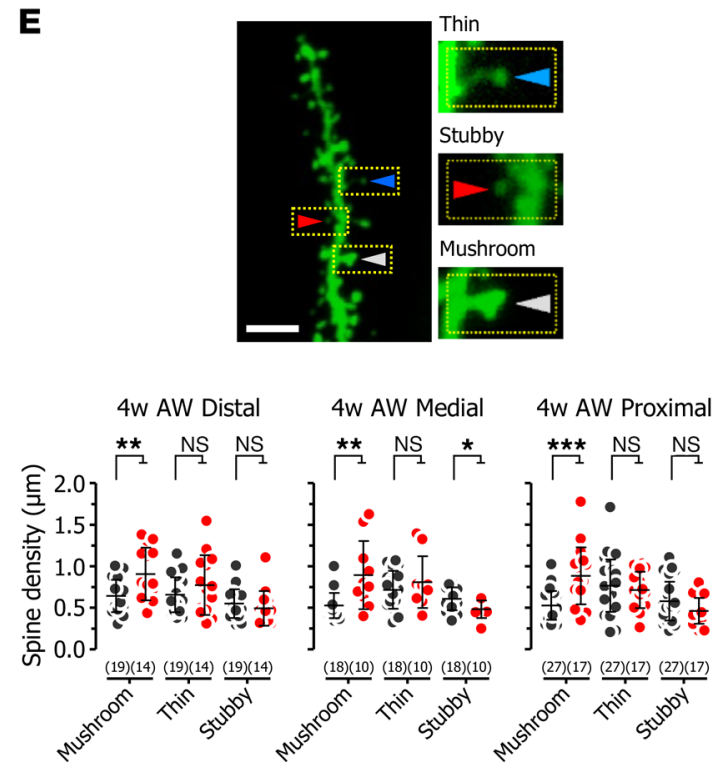

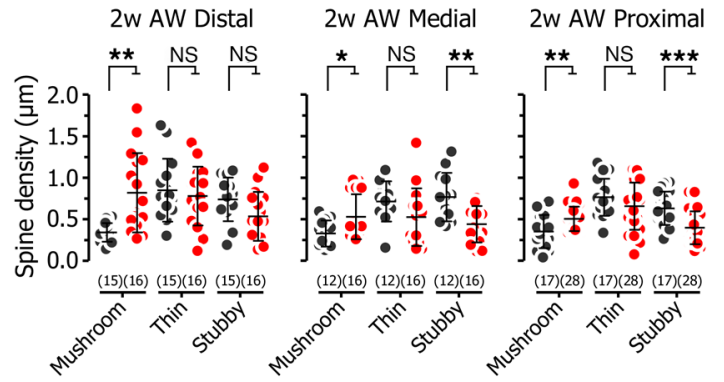

8W AW Distal

8w AW Medial $8 w$ AW Proximal

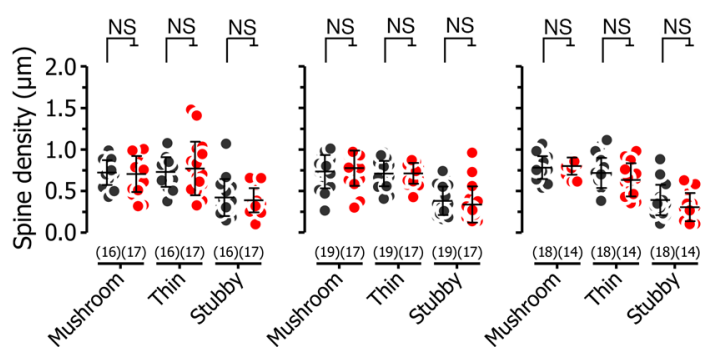

Figure 3. Alcohol withdrawal increases dendritic spine density of newborn dentate granule cells (DCCs) during abstinence. (A) Diagram showing the experimental schedule. A representative image shows GFP-expressing newborn DGCs. Z-stacked confocal images are projected into 2-dimensional images to display the morphology of GFP-expressing newborn DGCs in control (blue) and alcohol-fed mice (red). (B) The total dendritic length and number of dendritic branches of newborn DGCs were unchanged during abstinence (2-tailed unpaired $t$ tests). (C) An image shows the topological innervation of excitatory neurons into newborn DGCs (left). Representative images show spines located at the proximal, middle, and distal dendritic segments of newborn DGCs at 2 , 4 , and 8 weeks of abstinence (right). (D) Dendritic spine density of newborn DGCs was increased along the dendrites at 2 and 4 weeks of abstinence, but not at 8 weeks. (E) Densities of mushroom, stubby, and thin spines were analyzed in the distal, medial, and proximal dendritic segments of newborn DGCs at 2 , 4 , and 8 weeks of abstinence. ${ }^{*} P<0.05$; ${ }^{* *} P<0.01 ;{ }^{* *} P<0.001$ determined by 2 -tailed unpaired $t$ tests. 
A

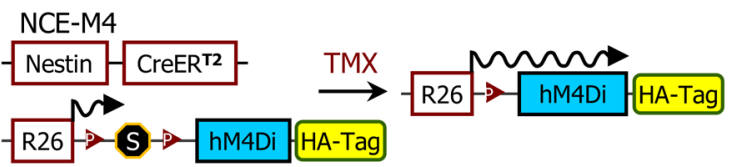

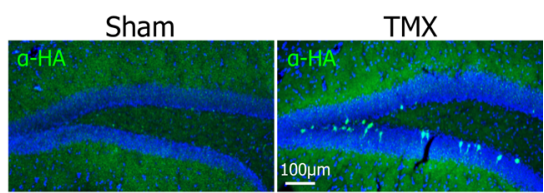

B

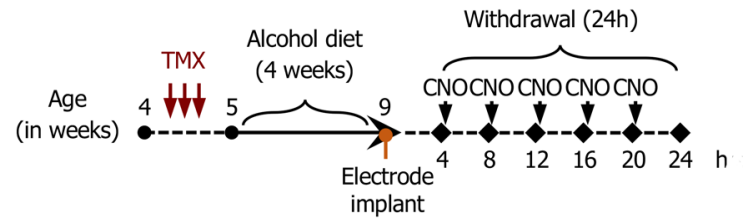

C

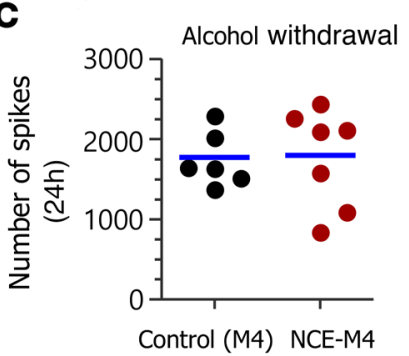

D
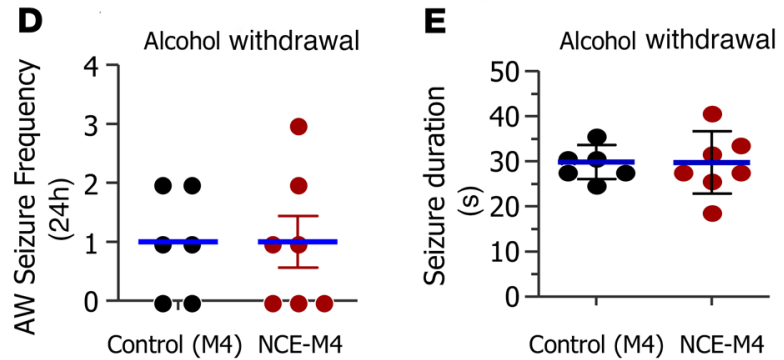

Figure 4. Chemogenetic inhibition of newborn DCCs reduces epileptiform SWDs during abstinence. (A) Experimental schedule examining the effects of specific inhibition of newborn DCCs on epileptiform SWDs during abstinence (left). $\mathrm{IHC}$ against an HA tag shows the expression of M4Di receptors in the dentate gyrus of the hippocampus (right). (B) Experimental timeline describing alcohol feeding and EEG recording schedule. CNO administration did not change the frequency of epileptic spikes (C), as well as seizure frequency (D) and duration (E) during the first 24 hours of AW. (F) Specific inhibition of newborn DGCs by the DREADD method decreased the frequency of epileptiform SWDs at 2, 3, and 4 weeks of abstinence. The effects of CNO on the expression of epileptiform SWDs were determined by 2-way repeated measures ANOVA with Bonferroni multiple comparison corrections. ${ }^{* *} P<0.001$.

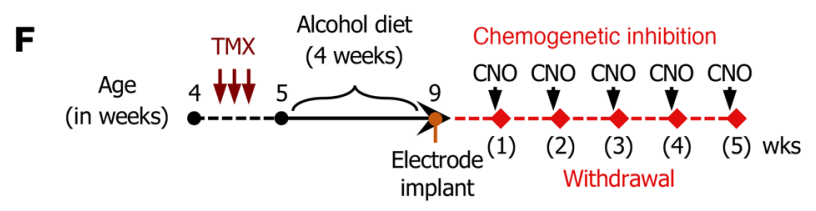

2w AW

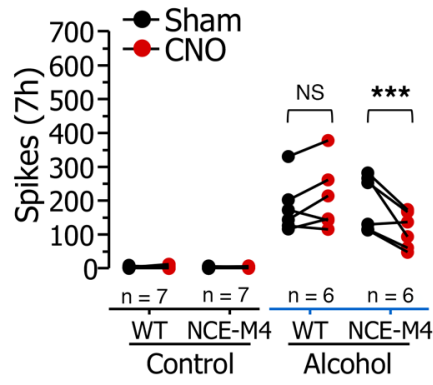

4 WW

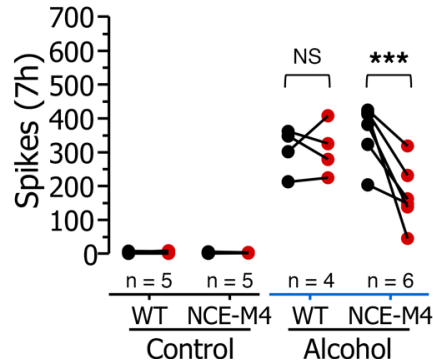

3w AW

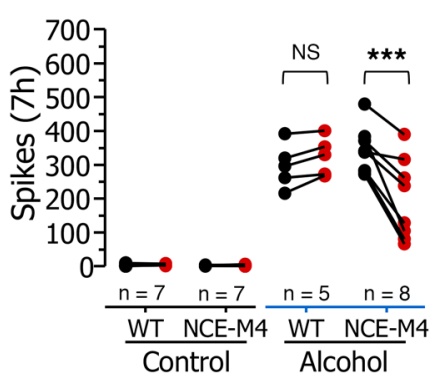

$5 \mathrm{~W}$ AW

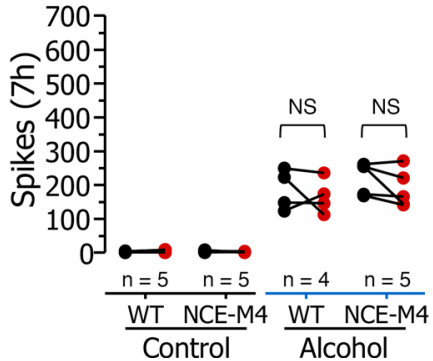

emotional stability (40-44), as well as neuropathological conditions such as seizures $(32,45,46)$. Indeed, our study showed that chronic alcohol exposure and withdrawal distinctly affect development and function of newborn DGCs, and as a result, newborn DGCs contribute to the production of phenotypes associated with alcohol exposure and withdrawal. While our previous study showed that chronic alcohol exposure significantly decreased the levels of neurogenesis and dendritic spine density of newborn DGCs (27), our current 

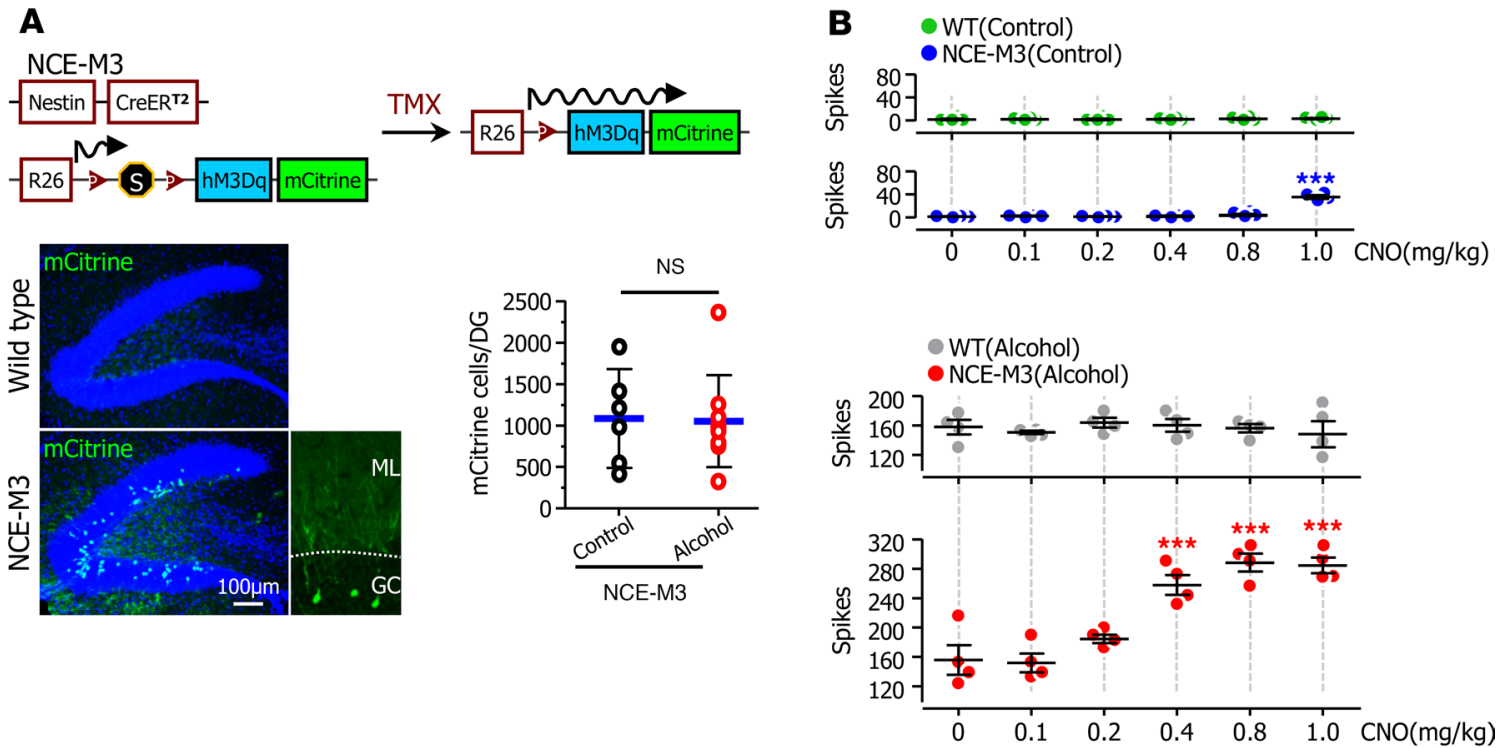

C

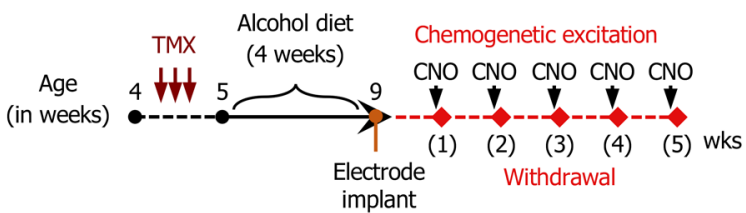

2w AW

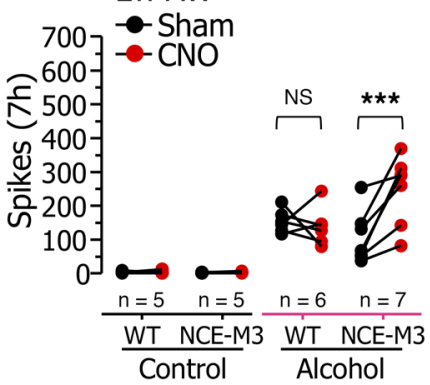

4w AW

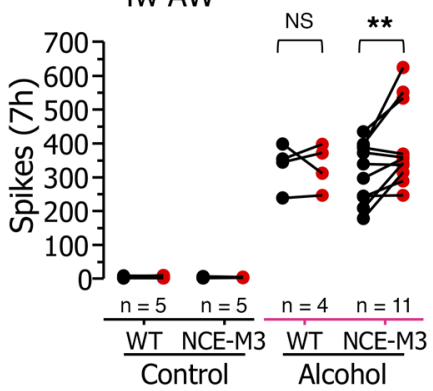

3w AW

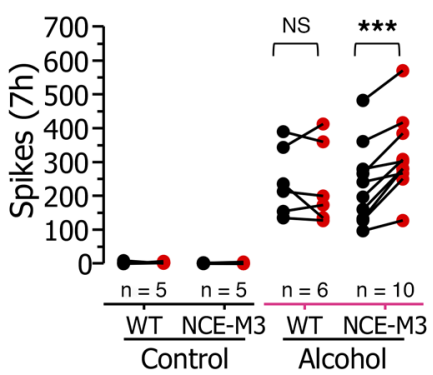

$5 \mathrm{~W} \mathrm{AW}$

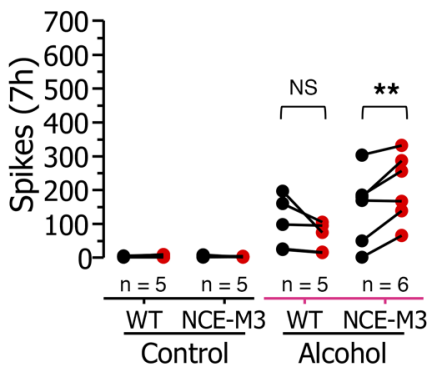

Figure 5. Chemogenetic activation of newborn DGCs increases epileptiform SWDs during abstinence. (A) Experimental schedule to determine the effect of specific activation of newborn DCCs on epileptiform SWDs during abstinence (top). IHC again mCitrine shows the expression of hM3Dq receptors in the dentate gyrus of the hippocampus in NCE-M3 mice (bottom). (B) A titration and determination of the precise dose of CNO that induces spikes only in NCE-M3 mice in an alcohol group. (C) Specific activation of newborn DGCs via the DREADD method increased the frequency of epileptiform SWDs at 2, 3, 4, and 5 weeks of abstinence, while control mice that were not exposed to alcohol or do not express excitatory hM3Dq receptors in newborn DGCs were not affected by CNO administration. The effects of CNO on the expression of epileptiform SWDs were determined by 2 -way repeated measures ANOVA with Bonferroni multiple comparison corrections. ${ }^{* *} P<0.01 ;{ }^{* *} P<0.001$. 
study demonstrates that AW increased neurogenesis (Supplemental Figure 5) and dendritic spine density of hippocampal DGCs, but minimally affected gliogenesis (Supplemental Figure 6), during a protracted abstinence. Thus, our study indicates that alcohol exposure and withdrawal distinctly induce synaptic connectivity of hippocampal newborn DGCs and that this decreased and increased synaptic connectivity of hippocampal newborn DGCs with excitatory neurons may contribute to depression and hyperexcitability of the brain during alcohol exposure and withdrawal.

In this study, we identified 2 distinct waves of development and progression of seizures during abstinence after chronic alcohol exposure. The first wave consists of a surge of multiple seizures and epileptic spikes within hours to days after AW. This first wave represents the symptoms of most alcohol abuse patients who are hospitalized and receive emergency treatment to control seizures and/or delirium tremens, the occurrence of which was reported to be as high as $15 \%$ of alcohol use-disorder patients. Our study shows that the first wave of AW seizures was independent of the activity of hippocampal newborn DGCs. It is plausible to reason that the allostatic state induced by chronic alcohol exposure was expressed in the form of the first wave of seizures upon a sudden termination of alcohol exposure. Interestingly, we found that AW seizures were sustained for a more protracted period of abstinence than has previously been reported. As the first wave of seizures diminished within a few days after AW, epileptiform SWDs started to appear at 1 week of abstinence, gradually increased and climaxed at 4 weeks of abstinence, and then decreased and were abolished by 6 weeks of abstinence. Compared with the first wave of AW seizures, only a few seizures were observed during the second wave of AW seizures. This progression of the second wave of seizures coincided with changes in dendritic spines of newborn DGCs during abstinence. The dendritic spines are the anatomical structures where excitatory synapses are formed on the dendrites of newborn DGCs, and lateral entorhinal, medial entorhinal, and hilar mossy cells project to the distal, medial, and proximal portion of dendrites of newborn DGCs in a topological manner. Our structural analysis revealed that AW globally increased the spine densities in all 3 dendritic segments of newborn DGCs. Moreover, the densities of mushroom spines increased at the expense of stubby spines, suggesting that AW promoted the transition of stubby spines into mushroom spines. Thus, AW globally induced synaptic connectivity of excitatory neurons onto hippocampal newborn DGCs, leading to AW-associated seizures during abstinence, and the increase or decrease in spine density of hippocampal newborn DGCs is tightly correlated with the severity of AW seizures during abstinence.

Interestingly, alcohol exposure and withdrawal did not change the global structure of hippocampal newborn DGCs, such as the total length of dendrites or the number of branches that determine the receptive field, but rather specifically impacted the density of dendritic spines. This may be because AW induces synaptic connectivity of excitatory input neurons onto hippocampal newborn DGCs, thus enhancing the excitability of newborn DGCs. Alternatively, AW may enhance the activity of hippocampal newborn DGCs, and this increased activity may induce synaptic redistribution from mature DGCs to newborn DGCs (47, 48). Although it is not clear whether the structural and functional AW-induced changes are intrinsic or extrinsic to newborn DGCs or both, our DREADD-mediated functional analysis clearly demonstrated that the activity of hippocampal newborn DGCs directly underlies AW seizures.

The DREADD method allowed us to manipulate the neuronal activity of hippocampal DGCs in an inducible and reversible manner at the moment when mice exhibited AW seizures during abstinence. Using this method, we showed that the specific activation and suppression of neuronal activity of hippocampal newborn DGCs increased and decreased the frequency of epileptiform SWDs, respectively, indicating that the activity of hippocampal newborn DGCs directly controls the expression of epileptiform SWDs during abstinence. A cell ablation method has often been used to determine the essential role of hippocampal newborn neurons in cognitive function and physiological excitability; however, this ablation method can induce network rearrangement of existing neurons, which makes it difficult to distinguish the direct role of newborn DGCs from the secondary effects of rearranged hippocampal networks $(47,49-52)$. Therefore, this inducible and reversible manipulation of neuronal activity allowed us to assess the necessity and sufficiency of hippocampal newborn DGCs in the expression of epileptiform SWDs when mice displayed AW seizures, and we were able to do so without inducing secondary network rearrangement. Of note, a recent study raised the possibility that clozapine can be converted from CNO. Clozapine is a potent antipsychotic drug that has numerous endogenous receptors, it readily penetrates the blood-brain barrier, and it potently binds DREADDs more than CNO (53). Therefore, it is critical to rule out potential nonspecific effects of CNO and/or side effects of clozapine metabolized from CNO. In the current study, we identified CNO-DREADD-specific effects by comparing CNO effects in both DREADD-expressing and non-DREADD-expressing mice in 
alcohol-fed as well as pair-fed groups (Figures 4 and 5 and refs. 54, 55). In addition, our CNO dose titration experiment indicated the induction of epileptiform SWDs specifically by CNO only in alcohol-fed mice. Thus, our $2 \times 2$ experimental design allowed us to analyze the specific effects of newborn DGCs on AW seizures, ruling out the potential involvement of nonspecific effects of DREADD and/or CNO.

In summary, our study demonstrated that the structural and functional changes in newborn DGCs during alcohol exposure and withdrawal are responsible for the expression of seizures during alcohol abstinence. This observation supports the hypothesis that neuroadaptation occurring during alcohol exposure may induce an allostatic state in the brain and that the expression of this allostatic state may underlie AWS. In line with this hypothesis, our study proposes that dynamic changes in plasticity of hippocampal newborn DGCs directly contribute to neuroadaptation that is essential for the expression of phenotypes associated with alcohol exposure and withdrawal. Thus, our study provides important insight into hippocampal newborn DGCs that can be used to intervene and/or treat AW seizures.

\section{Methods}

Supplemental Methods are available online with this article.

Animals. Four-week-old mice were used in this study. DREADD_hM3Dq ${ }^{\mathrm{fl} / \mathrm{fl}}(\mathrm{M} 3)$ mice (stock no. 026220) (33) and C57BL/6 (stock no. 000664) mice were purchased from The Jackson Laboratory. Nestin-CreER ${ }^{\mathrm{T} 2}$ and DREADD_hM4Dit/fll (M4) mice were provided by Amelia Eisch (56) (University of Pennsylvania, Philadelphia, Pennsylvania, USA) and Susan Dymecki (31) (Harvard Medical School, Boston, Massachusetts, USA), respectively. All transgenic mice were backcrossed to C57BL/ 6 for more than 10 generations.

Data acquisition and analysis. To reduce bias in data collection, analysis, and reporting, experimental conditions and subjects were randomly coded, and the investigators were blinded to treatment conditions and codes until all data had been collected and analyzed.

Tamoxifen and CNO treatment to express and activate DREADD receptors. To express hM3Dq or hM4Di receptors in newborn DGCs, tamoxifen $(180 \mathrm{mg} / \mathrm{kg}$, i.p., dissolved in a 1:10 mixture of ethanol and corn oil, MilliporeSigma) was administered to Nestin-CreER ${ }^{\mathrm{T} 2} ; \mathrm{DREADD}$ hM3Dq ${ }^{\mathrm{f} / \mathrm{fl}}$ (NCE-M3) or Nestin-CreER ${ }^{\mathrm{T} 2}$;DREADD_hM4Di ${ }^{\mathrm{fl} / \mathrm{fl}}$ (NCE-M4) double transgenic mice for 5 days when the mice were 4 weeks old. CNO (MilliporeSigma) was first dissolved in DMSO and then brought to final concentration with $0.9 \%$ saline and a final concentration of DMSO of $0.5 \%$ (MilliporeSigma). Doses of 0.8 or $10 \mathrm{mg} / \mathrm{kg}$ of CNO were injected into NCE-M3 or NCE-M4 mice, respectively.

A pair-fed chronic alcohol exposure mouse model. Mice had voluntary access to a nutritionally adequate liquid diet containing either ethanol or dextrose. In 1 serving of the Lieber-DeCarli control diet (Dyets Inc.), $18 \%$ of energy is derived from protein, $35 \%$ from fat, and $47 \%$ from carbohydrate (27). In the alcohol diet ( $5 \%$ ethanol, v/v), ethanol provided $27 \%$ of dietary energy. In the control diet, ethanol was replaced by an isocaloric amount of maltodextrin (BioServ; F1697SP). Five-week-old, weight-matched mice were randomly distributed into pair-fed control and alcohol-fed experimental groups and consumed either control or alcohol-containing food for 4 weeks.

IHC. Animals were deeply anesthetized with a cocktail of ketamine (400 mg/kg) and xylazine (40 mg/ $\mathrm{kg}$ ) and transcardially perfused first with phosphate buffer and subsequently with $4 \%$ paraformaldehyde (PFA). Brains were dissected and postfixed overnight in PFA at $4^{\circ} \mathrm{C}$. Brains were cryoprotected in $30 \%$ sucrose for 48 hours at $4^{\circ} \mathrm{C}$ and sectioned at $40 \mu \mathrm{m}$ with a sliding microtome (Leica SM2010 R). Floating tissue sections were washed 3 times in tris-buffered saline (TBS) and permeabilized in $0.3 \%$ Triton X-100 (TBST) (MilliporeSigma) for 30 minutes. Tissues were blocked in TBST containing 3\% donkey serum and then incubated with primary antibodies (chicken anti-GFP, 1:400, GFP-1010, Aves Labs) overnight at $4^{\circ} \mathrm{C}$. Tissues were then washed 3 times for 10 minutes in TBST before incubation with secondary antibodies at 1:400 (donkey anti-chicken; Alexa Fluor 488, catalog 703-545-155, Jackson ImmunoResearch) for 2 hours. Tissues were then washed 3 times in TBST for 10 minutes. Finally, tissues were rinsed with TBS containing $500 \mathrm{nM}$ DAPI for 10 minutes and then with a final wash in TBS for 10 minutes. Tissues were then mounted on slides and cover-slipped.

Morphological analysis of GFP-labeled newborn DGCs and morphometric analysis of dendritic spines. Imaging and analysis of the general morphology of GFP-labeled newborn DGCs have been previously described (27). Briefly, for morphological analysis, z-series images with $1-\mu \mathrm{m}$ intervals were acquired using a Leica confocal microscope with a 40× oil lens (SP5; Leica), and total dendritic length and the number of dendritic branching points were analyzed using IMARIS software (Bitplane). Maximal projected, 2-dimensional images were presented in line images. For measurement of spine densities, the outer-, middle-, and inner-third of dendrites of GFP-labeled 
newborn neurons were imaged with a confocal microscope under the following conditions: $63 \times$ oil lens, digital zoom factor 5 , and $0.1-\mu \mathrm{m}$ interval. Maximum intensity projection of these z-series images was produced, and the length of each dendritic segment was measured in Leica Image Software. Two-dimensional projection images were imported to Adobe Photoshop (Adobe Systems Inc.), and the number of spines was counted manually. To analyze spine densities in the distal, medial, and proximal dendritic segments of DGCs, more than 10 dendritic segments per group were imaged and reconstructed in ImageJ software (NIH). Then, spines were categorized into mushroom, thin, or stubby spines on the basis of 3 parameters, including the spine length, diameter of neck, and diameter of spine head $(28,57,58)$. Spines were scored as mushrooms if the diameter of the head was 3 times larger than that of the neck. We defined spines as stubby spines if the diameter of the spine head was greater than the spine length. Thin spines were scored when the length of spine was greater than the diameter of the head and the diameter of the head was greater than the diameter of the neck.

EEG recording. Animals were anesthetized with a mixture of ketamine (100 mg/kg, ACE Surgical Supply Co.) and xylazine (10 mg/kg, MilliporeSigma) and placed in a stereotactic apparatus (David Kopf Instruments). For hippocampal recordings, pedestal gold-plated stainless-steel electrodes ( $0.2 \mathrm{~mm}$ in diameter; PlasticsOne) were placed bilaterally in the intermediate regions of hippocampi $(\mathrm{LH}$, left hippocampus; $\mathrm{RH}$, right hippocampus; $2.48 \mathrm{~mm}$ posterior to the bregma, $2.63 \mathrm{~mm}$ lateral to the midline, and $3.38 \mathrm{~mm}$ below the dura). Stainless-steel screws (E363/96/2.4/SPC; PlasticsOne) were placed on the dura mater bilaterally over frontal motor cortex (LC, left frontal; RC, right frontal, $0.5 \mathrm{~mm}$ posterior to the bregma, $2.45 \mathrm{~mm}$ lateral to the midline). An additional stainless-steel screw was placed just to the right of the frontal sinus and served as a reference electrode (59). Continuous EEG recoding with simultaneous video monitoring was performed. During the recording, mice were unrestrained in the monitoring boxes with access to food and water ad libitum. Although EEG was monitored and recorded for 16 hours, we used the first 8 hours of recording for the data analysis because the effect of 1 dose of CNO lasts for up to 8 hours. The sampling rate was $100 \mathrm{~Hz}$ with $5.3 \mathrm{~Hz}$ and $30 \mathrm{~Hz}$ filtering frequencies. Correlations between EEG events and overt behavioral responses were assessed.

EEG analysis. EEG spikes were defined as paroxysmal electrical sharp activity with durations of 20-150 ms, with amplitudes that were at least 2 times the background EEG activity. An in-house developed, MATLAB-based program was used for analysis of spike frequency, amplitude, and duration. A digital bandpass filter $(1-30 \mathrm{~Hz})$ was applied to attenuate high-frequency noise and to reduce low-frequency drift artifacts. A double-threshold algorithm was used to avoid counting small ripples as independent spikes; only spikes that passed both initiation threshold $(\mathrm{THi}=100 \mu \mathrm{V})$ and termination threshold $(\mathrm{THt}=0 \mu \mathrm{V})$ were counted (Supplemental Figure 2). The rates of occurrence of all ictal spikes were calculated each recording day. After EEG recording was completed, mice were sacrificed, and the location of hippocampal depth electrodes was confirmed with H\&E staining.

Statistics. Statistical analyses were performed using commercial software (GraphPad Prism; GraphPad Software, Inc). For all results, the significance threshold was placed at $\alpha=0.05\left({ }^{*} P<0.05 ;{ }^{*} P<0.01 ;{ }^{* *} P\right.$ $<0.001 ;{ }^{* * *} P<0.0001$ ), and corrections for multiple comparisons are reflected in the $P$ value rather than in $\alpha$. Correlational analyses were evaluated by using the Pearson's Correlation test. All data descriptions are shown as mean \pm SD and/or mean \pm SEM.

Study approval. All procedures involving animals were approved by the IACUC of Cleveland Clinic. All experiments were conducted in accordance with the principles and procedures outlined in the NIH Guide for the Care and Use of Laboratory Animals (National Academies Press, 2011).

\section{Author contributions}

DL and HS designed the research, analyzed the data, and wrote the manuscript. DL, HZ, HRP, EJR, and YNJ performed the research. BK performed the EEG statistical analyses. All authors contributed to analysis and discussion of the results. All authors read and approved the final manuscript.

\section{Acknowledgments}

We thank Christopher L. Nelson for editorial support. We also thank Timothy Hecker for technical support. This work was supported by the National Institute of Alcohol Abuse and Alcoholism (R01AA022377, to HS) and the Hartwell Foundation (to HS).

Address correspondence to: Hoonkyo Suh, Department of Neurosciences, NC 30, Cleveland Clinic, Cleveland, Ohio 44195, USA. Phone: 216.444.5895; Email: suhh2@ccf.org. 
1. Koob GF. Theoretical frameworks and mechanistic aspects of alcohol addiction: alcohol addiction as a reward deficit disorder. Curr Top Behav Neurosci. 2013;13:3-30.

2. Koob GF. Neurocircuitry of alcohol addiction: synthesis from animal models. Handb Clin Neurol. 2014;125:33-54.

3. Victor M. The alcohol withdrawal syndrome: theory and practice. Postgrad Med. 1970;47(4):68-72.

4. Becker HC. Animal models of alcohol withdrawal. Alcohol Res Health. 2000;24(2):105-113.

5. Becker HC. Alcohol dependence, withdrawal, and relapse. Alcohol Res Health. 2008;31(4):348-361.

6. Becker HC, Mulholland PJ. Neurochemical mechanisms of alcohol withdrawal. Handb Clin Neurol. 2014;125:133-156

7. McKeon A, Frye MA, Delanty N. The alcohol withdrawal syndrome. J Neurol Neurosurg Psychiatry. 2008;79(8):854-862.

8. Saitz R. Patients with alcohol problems. NEngl J Med. 1998;339(2):130-131.

9. Finn DA, Crabbe JC. Exploring alcohol withdrawal syndrome. Alcohol Health Res World. 1997;21(2):149-156.

10. Littleton J. Neurochemical mechanisms underlying alcohol withdrawal. Alcohol Health Res World. 1998;22(1):13-24.

11. Noël X, Bechara A, Dan B, Hanak C, Verbanck P. Response inhibition deficit is involved in poor decision making under risk in nonamnesic individuals with alcoholism. Neuropsychology. 2007;21(6):778-786.

12. Schuckit MA. Recognition and management of withdrawal delirium (delirium tremens). N Engl J Med. 2014;371(22):2109-2113

13. Maldonado JR, et al. The "Prediction of Alcohol Withdrawal Severity Scale" (PAWSS): systematic literature review and pilot study of a new scale for the prediction of complicated alcohol withdrawal syndrome. Alcohol. 2014;48(4):375-390.

14. Jesse S, et al. Alcohol withdrawal syndrome: mechanisms, manifestations, and management. Acta Neurol Scand. 2017;135(1):4-16

15. Koob GF, Le Moal M. Addiction and the brain antireward system. Annu Rev Psychol. 2008;59:29-53.

16. Koob GF. Alcoholism: allostasis and beyond. Alcohol Clin Exp Res. 2003;27(2):232-243.

17. Koob GF, Le Moal M. Drug addiction, dysregulation of reward, and allostasis. Neuropsychopharmacology. 2001;24(2):97-129.

18. Becker HC. Kindling in alcohol withdrawal. Alcohol Health Res World. 1998;22(1):25-33.

19. Brown ME, Anton RF, Malcolm R, Ballenger JC. Alcohol detoxification and withdrawal seizures: clinical support for a kindling hypothesis. Biol Psychiatry. 1988;23(5):507-514.

20. Monte R, Rabuñal R, Casariego E, López-Agreda H, Mateos A, Pértega S. Analysis of the factors determining survival of alcoholic withdrawal syndrome patients in a general hospital. Alcohol Alcohol. 2010;45(2):151-158.

21. Rogawski MA. Update on the neurobiology of alcohol withdrawal seizures. Epilepsy Curr. 2005;5(6):225-230.

22. Gonzalez LP, Veatch LM, Ticku MK, Becker HC. Alcohol withdrawal kindling: mechanisms and implications for treatment. Alcohol Clin Exp Res. 2001;25(5 Suppl ISBRA):197S-201S.

23. Sand T, Bjørk M, Bråthen G, Michler RP, Brodtkorb E, Bovim G. Quantitative EEG in patients with alcohol-related seizures. Alcohol Clin Exp Res. 2010;34(10):1751-1758.

24. Veatch LM, Gonzalez LP. Repeated ethanol withdrawal produces site-dependent increases in EEG spiking. Alcohol Clin Exp Res. 1996;20(2):262-267.

25. Hughes JR. Alcohol withdrawal seizures. Epilepsy Behav. 2009;15(2):92-97.

26. Lieber CS, DeCarli LM. Liquid diet technique of ethanol administration: 1989 update. Alcohol Alcohol. 1989;24(3):197-211.

27. Golub HM, et al. Chronic Alcohol Exposure is Associated with Decreased Neurogenesis, Aberrant Integration of Newborn Neurons, and Cognitive Dysfunction in Female Mice. Alcohol Clin Exp Res. 2015;39(10):1967-1977.

28. Zhao C, Teng EM, Summers RG, Ming GL, Gage FH. Distinct morphological stages of dentate granule neuron maturation in the adult mouse hippocampus. J Neurosci. 2006;26(1):3-11.

29. Amaral DG, Scharfman HE, Lavenex P. The dentate gyrus: fundamental neuroanatomical organization (dentate gyrus for dummies). Prog Brain Res. 2007;163:3-22.

30. Rogan SC, Roth BL. Remote control of neuronal signaling. Pharmacol Rev. 2011;63(2):291-315.

31. Ray RS, et al. Impaired respiratory and body temperature control upon acute serotonergic neuron inhibition. Science. 2011;333(6042):637-642.

32. Zhou QG, et al. Chemogenetic silencing of hippocampal neurons suppresses epileptic neural circuits. J Clin Invest. 2019;129(1):310-323.

33. Zhu H, et al. Cre-dependent DREADD (Designer Receptors Exclusively Activated by Designer Drugs) mice. Genesis. 2016;54(8):439-446.

34. Crews FT, et al. Alcoholic neurobiology: changes in dependence and recovery. Alcohol Clin Exp Res. 2005;29(8):1504-1513

35. Sullivan EV, Rosenbloom MJ, Lim KO, Pfefferbaum A. Longitudinal changes in cognition, gait, and balance in abstinent and relapsed alcoholic men: relationships to changes in brain structure. Neuropsychology. 2000;14(2):178-188.

36. George O, Koob GF, Vendruscolo LF. Negative reinforcement via motivational withdrawal is the driving force behind the transition to addiction. Psychopharmacology (Berl). 2014;231(19):3911-3917.

37. Suh H, Deng W, Gage FH. Signaling in adult neurogenesis. Annu Rev Cell Dev Biol. 2009;25:253-275.

38. Suh H, Consiglio A, Ray J, Sawai T, D’Amour KA, Gage FH. In vivo fate analysis reveals the multipotent and self-renewal capacities of Sox2+ neural stem cells in the adult hippocampus. Cell Stem Cell. 2007;1(5):515-528.

39. Ming GL, Song H. Adult neurogenesis in the mammalian brain: significant answers and significant questions. Neuron. 2011;70(4):687-702.

40. Sahay A, Wilson DA, Hen R. Pattern separation: a common function for new neurons in hippocampus and olfactory bulb. Neuron. 2011;70(4):582-588.

41. Sahay A, et al. Increasing adult hippocampal neurogenesis is sufficient to improve pattern separation. Nature. 2011;472(7344):466-470.

42. Clelland CD, et al. A functional role for adult hippocampal neurogenesis in spatial pattern separation. Science. 2009;325(5937):210-213.

43. Gilbert PE, Kesner RP, DeCoteau WE. Memory for spatial location: role of the hippocampus in mediating spatial pattern separation. J Neurosci. 1998;18(2):804-810.

44. Santarelli L, et al. Requirement of hippocampal neurogenesis for the behavioral effects of antidepressants. Science. 2003;301(5634):805-809.

45. Cho KO, et al. Aberrant hippocampal neurogenesis contributes to epilepsy and associated cognitive decline. Nat Commun. 
$2015 ; 6: 6606$.

46. Hosford BE, Liska JP, Danzer SC. Ablation of Newly Generated Hippocampal Granule Cells Has Disease-Modifying Effects in Epilepsy. J Neurosci. 2016;36(43):11013-11023.

47. Adlaf EW, et al. Adult-born neurons modify excitatory synaptic transmission to existing neurons. Elife. 2017;6:e19886.

48. McAvoy KM, et al. Modulating Neuronal Competition Dynamics in the Dentate Gyrus to Rejuvenate Aging Memory Circuits. Neuron. 2016;91(6):1356-1373.

49. Singer BH, Gamelli AE, Fuller CL, Temme SJ, Parent JM, Murphy GG. Compensatory network changes in the dentate gyrus restore long-term potentiation following ablation of neurogenesis in young-adult mice. Proc Natl Acad Sci USA. 2011;108(13):5437-5442.

50. Yasuda M, Johnson-Venkatesh EM, Zhang H, Parent JM, Sutton MA, Umemori H. Multiple forms of activity-dependent competition refine hippocampal circuits in vivo. Neuron. 2011;70(6):1128-1142.

51. Burghardt NS, Park EH, Hen R, Fenton AA. Adult-born hippocampal neurons promote cognitive flexibility in mice. Hippocampus. 2012;22(9):1795-1808.

52. Lacefield CO, Itskov V, Reardon T, Hen R, Gordon JA. Effects of adult-generated granule cells on coordinated network activity in the dentate gyrus. Hippocampus. 2012;22(1):106-116.

53. Gomez JL, et al. Chemogenetics revealed: DREADD occupancy and activation via converted clozapine. Science. 2017;357(6350):503-507.

54. Mahler SV, Aston-Jones G. CNO Evil? Considerations for the Use of DREADDs in Behavioral Neuroscience. Neuropsychopharmacology. 2018;43(5):934-936.

55. Smith KS, Bucci DJ, Luikart BW, Mahler SV. DREADDS: Use and application in behavioral neuroscience. Behav Neurosci. 2016;130(2):137-155.

56. Lagace DC, et al. Dynamic contribution of nestin-expressing stem cells to adult neurogenesis. J Neurosci. 2007;27(46):12623-12629.

57. Arellano JI, Benavides-Piccione R, Defelipe J, Yuste R. Ultrastructure of dendritic spines: correlation between synaptic and spine morphologies. Front Neurosci. 2007;1(1):131-143.

58. Qiao H, Li MX, Xu C, Chen HB, An SC, Ma XM. Dendritic Spines in Depression: What We Learned from Animal Models. Neural Plast. 2016;2016:8056370.

59. Kellinghaus C, et al. Dissociation between in vitro and in vivo epileptogenicity in a rat model of cortical dysplasia. Epileptic Disord 2007;9(1):11-19. 